\title{
FUZZY CLUSTERING METHODS FOR OBJECT LOCALIZATION
}

\section{Raghu Krishnapuram, European Laboratory for Intelligent Techniques Engineering, Aachen}

Object localization (i. e., determination of the location and orientation of objects) plays an important role in many computer vision applici tions such as automated assembly and target tracking. In this paper, we consider the problem of object localization when the object model is known. It is assumed that the object boundary (in the case of two-dimensional objects and intensity images) or the object surface (in the case of three-dimensional objects and range images) can be represented by a polynomial of arbitrary degree. However, the number of objects present in the image may or may not be known, and some of the objects may be occluded.

Fuzzy objective function based clustering methods have been used extensively for pattern recognition and computer vision. The Fuzzy C Means (FCM) algorithm due to Bezdek, and its derivatives have been used to detect clusters of various shapes. More recently, this approach has been extended to the case of shell clusters, i. e., clusters with no interior points. The boundary or the surface of the clusters is assumed to be quadric (second-degree) in shape. The prototypes of the clusters are represented by a parameter vector, which consists of the coefficients of the quadric. In shell clustering algorithms, the parameter vector is assumed to be unknown. However, in the present application, we assume that the curve or surfare parameters are known, but only the translation and rotation parameters are to be determined. To achieve this, we construct a suitable fuzzy objective function, and design a fuzzy clustering algorithm based on the objective function to determine the rotation matrices and translation vectors for all the objects in the image.

\footnotetext{
* On leave from: The Department of Electrical and Computer Engineering, University of Missouri, Columbia, MO 65211, USA.
} 